\title{
Statistical properties of modal noise in fiber-laser systems
}

\author{
B. Crosignani* and A. Yariv \\ Department of Applied Physics, California Institute of Technology, Pasadena, California 91125
}

Received November 4, 1982

\begin{abstract}
The direct analysis in the time domain of the fluctuations of a signal propagating in a fiber-optic link in the presence of an imperfect connector makes it possible to formalize in a simple manner the description of its statistical properties. This permits, in particular, the clarification of the role played by the various time scales involved in the problem (coherence time of the fiber-exciting source, fiber modal delay, detector response time, etc.) in evaluating the statistical averages. The formalism includes in a straightforward way the case of simultaneous excitation of the fiber by more than one source. This last circumstance is expedient for checking the beneficial effect on modal noise derived from exciting the fiber with $N$ laser sources.
\end{abstract}

\section{INTRODUCTION}

The characteristics of the speckle pattern appearing on the end face of a multimode optical fiber within which partially coherent radiation propagates are influenced by the coherence time of the exciting source and the relative time delays among the various fiber modes. ${ }^{1,2}$ The fact that different speckle patterns exhibit different transmission losses when a connector is inserted between two fibers has been recognized as an important possible source of noise in fiber communication links (modal noise). ${ }^{3}$ To assist in understanding the effect quantitatively, a number of investigations of speckle patterns as they appear on a fiber exit face have been reported. Since such speckle patterns are usually time varying at random because of fluctuations both in the exciting source and in the fiber environment, statistical methods have quite naturally been used to advantage in the calculation of the ensemble average (equivalent to time averaging over a long time interval under the ergodicity hypothesis) of some important relevant statistical quantities, such as average power and the variance. ${ }^{4-6}$ More recently, the problem of noise in optical communication systems that is due to fiber connectors was considered by Petermann ${ }^{7}$ in a more direct way by introducing the spectral coupling efficiency $\eta(\omega)$ of the connector, a quantity whose statistical fluctuations are a measure of the noise and distortion characteristics of the connector.

This last approach, although it is straightforward and useful from the point of view of system engineering, does not lend itself easily to clarifying the role played by the various time constants involved in the problem, especially when more than one source is employed for exciting the fiber. This has led us to adopt a complementary approach in the time domain, where the various time scales appear in an explicit way and the processing of the signal leading to the evaluation of the statistical averages characterizing modal noise is better understood. In particular, we evaluate the variance of the signal power following the connector for various situations, including those pertaining to the case in which the fiber is simultaneously excited by many sources (or by the various longitudinal modes of a single-mode laser source).

\section{PROPAGATION THROUGH THE FIBER AND THE CONNECTOR}

We adopt in the following the same schematic configuration for the fiber and the connector as in Ref. 7 (see Fig. 1), in which the fibers are assumed to be butt jointed, with no relative tilt, and the reflections at the fiber connector are neglected. The transverse part of the analytic signal of the electric field in fiber I can then be written as a superposition of guided modes in the form 8

$$
\begin{aligned}
& \hat{\mathbf{E}}^{(\mathrm{I})}(\mathbf{r}, z, t)=\sum_{n} \mathbf{E}_{n}^{(\mathrm{I})}(\mathbf{r}) \sum_{\mu} \exp \left[i \omega_{\mu} t-i \beta_{n}\left(\omega_{\mu}\right) z\right] \\
& \times A_{n}{ }^{(\mu)}(t) \Phi_{n}{ }^{(I)}(\mu)\left(t-z / V_{n}\right),
\end{aligned}
$$

where the Latin indices label the fiber modes (whose propagation constants and spatial mode configurations are indicated, respectively, by $\beta_{m}$ and $\mathbf{E}_{m}$ ) and the Greek indices indicate the different sources (each centered at its own angular frequency $\omega_{\mu}$ ). In Eq. (1) we have neglected fiber mode coupling, chromatic dispersion, and the dependence of the mode profile on the frequency. We have indicated by $V_{n}$ the group velocity of the $n$th mode,

$$
V_{n}=\left(\frac{\mathrm{d} \beta_{n}}{\mathrm{~d} \omega}\right)_{\omega=\omega_{0}}^{-1},
$$

where $\omega_{0}$ is an arbitary midfrequency of the total field whose bandwidth $\delta \omega$ is such that

$$
\frac{\delta \omega}{\omega_{0}} \ll 1
$$

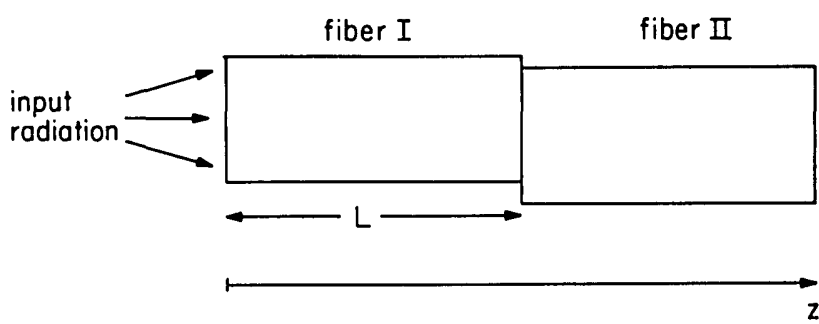

Fig. 1. Schematic configuration of the source-fiber-connector system. 
The complex amplitudes $A_{n}{ }^{(\mu)}(t)$ exhibit a time dependence associated with the slow time variations of the fiber environment, whereas the $\Phi^{(\mathrm{I})(\mu)}(t)$ 's contain the time dependence (which is due to both modulation and noise) of the $\mu$ th exciting source.

After the electric field in fiber II is written as the sum of a guided part $\hat{\mathbf{E}}_{G}{ }^{(\mathrm{II})}$ and a radiation part $\hat{\mathbf{E}}_{R}$ (II),

$$
\begin{aligned}
\hat{\mathbf{E}}^{(\mathrm{II})}(\mathbf{r}, z, t)= & \hat{\mathbf{E}}_{R}{ }^{(\mathrm{II})}+\hat{\mathbf{E}}_{G}^{(\mathrm{II})} \\
= & \hat{\mathbf{E}}_{R}^{(\mathrm{II})}+\sum_{m} \mathbf{E}_{m}^{(\mathrm{II})}(\mathbf{r}) \sum_{\mu} \exp \left[i \omega_{\mu} t-i \beta_{m}\left(\omega_{\mu}\right)\right. \\
& \times(z-L)] B_{m}^{(\mu)} \Phi_{m}^{(\mathrm{II})(\mu)}\left(t-\frac{z-L}{V_{m}}\right)
\end{aligned}
$$

the matching of the two fields at $z=L, \hat{\mathbf{E}}^{(\mathrm{I})}(\mathbf{r}, z=L, t)=$ $\hat{\mathbf{E}}^{(\mathrm{II})}(\mathbf{r}, z=L, t)$ yields, taking advantage of the orthogonality condition of the guided modes (among themselves and with the radiation modes),

$$
\begin{aligned}
& B_{m}{ }^{(\mu)} \Phi_{m}{ }^{(\mathrm{II})(\mu)}(t)=\sum_{n}\left(\iint \mathbf{E}_{n}{ }^{(\mathrm{I})}(\mathbf{r}) \times \mathbf{H}_{m}{ }^{(\mathrm{II})^{*}}(\mathbf{r}) \cdot \hat{z} \mathrm{~d} \mathbf{r}\right) \\
& \quad \times \exp \left[-i \beta_{n}\left(\omega_{\mu}\right) L\right] A_{n}{ }^{(\mu)} \Phi_{n}{ }^{(\mathrm{I})(\mu)}\left(t-L / V_{n}\right),
\end{aligned}
$$

where $\mathbf{H}_{m}$ denotes the magnetic field of the $m$ th mode and the integration over the transverse coordinate $\mathbf{r}$ extends over the whole fiber section. We can then write, limiting ourselves to the field just past the connector,

$$
\hat{\mathbf{E}}_{G}^{(\mathrm{II})}(\mathbf{r}, L, t)=\sum_{\mu} \sum_{m} \mathbf{E}_{m}^{(\mathrm{II})}(\mathbf{r}) B_{m}^{(\mu)} \exp \left(i \omega_{\mu} t\right),
$$

where

$$
\begin{aligned}
& B_{m}{ }^{(\mu)}=\sum_{n} I_{n m}{ }^{(\mu)} A_{n}{ }^{(\mu)} \Phi_{n}{ }^{(\mu)}\left(t-\frac{L}{V_{n}}\right), \\
I_{n m}{ }^{(\mu)}= & {\left[\iint \mathbf{E}_{n}{ }^{(\mathrm{I})} \times \mathbf{H}_{m}{ }^{(\mathrm{II})^{*}} \cdot \hat{z} \mathrm{~d} \mathbf{r}\right] \exp \left[-i \beta_{n}\left(\omega_{\mu}\right) L\right] } \\
\equiv & \mathcal{J}_{n m} \exp \left[-i \beta_{n}\left(\omega_{\mu}\right) L\right], \\
& \Phi_{n}{ }^{(\mu)}\left(t-\frac{L}{V_{n}}\right) \equiv \Phi_{n}{ }^{(\mathrm{I})(\mu)}\left(t-\frac{L}{V_{n}}\right) .
\end{aligned}
$$

The total power carried by the fiber past the connector is then proportional to

$$
P(t)=\sum_{m}\left|\sum_{\mu} B_{m}{ }^{(\mu)} \exp \left(i \omega_{\mu} t\right)\right|^{2},
$$

which can be written, after defining

$$
\begin{aligned}
\mathcal{F}_{n k}{ }^{(\mu, \nu)} & =\sum_{m} I_{n m}{ }^{(\mu)} I_{k m}{ }^{(\nu) *} \\
& =\left(\sum_{m} \mathfrak{I}_{n m} \mathfrak{I}_{k m} *\right) \exp \left[i \beta_{k}\left(\omega_{\nu}\right) L-i \beta_{n}\left(\omega_{\mu}\right) L\right],
\end{aligned}
$$

in the form

$$
\begin{aligned}
P(t)= & \sum_{n} \sum_{k} \sum_{\mu} \sum_{\nu} \exp \left[i\left(\omega_{\mu}-\omega_{\nu}\right) t\right] \\
& \times \mathscr{F}_{n k}{ }^{(\mu, \nu)} A_{n}{ }^{(\mu)} A_{k}{ }^{(\nu) *} \Phi_{n}{ }^{(\mu)} \\
& \times\left(t-\frac{L}{V_{n}}\right) \phi_{k}{ }^{(\nu) *}\left(t-\frac{L}{V_{k}}\right) .
\end{aligned}
$$

In particular, if only one source is employed $\left(\omega_{\mu}=\omega_{\nu}=\ldots\right.$. $\left.=\omega_{0}\right)$, Eq. $(12)$ reduces to

$$
P(t)=\sum_{n} \sum_{k} \mathcal{F}_{n k} A_{n} A_{k} * \Phi_{n}\left(t-\frac{L}{V_{n}}\right) \Phi_{k} *\left(t-\frac{L}{V_{k}}\right),
$$

with

$$
\mathscr{F}_{n k}=\left(\sum_{m} \mathcal{I}_{n m} \mathcal{I}_{k m} *\right) \exp \left[i \beta_{k}\left(\omega_{0}\right) L-i \beta_{n}\left(\omega_{0}\right) L\right]=\mathscr{F}_{k n} *
$$

At this point it is worthwhile to note that it is usually possible to write

$$
\Phi_{n}^{(\mu)}\left(t-\frac{L}{V_{n}}\right)=\Phi^{(\mu)}\left(t-\frac{L}{V_{n}}\right),
$$

that is, all the modes of $\omega_{\mu}$ are excited initially in a similar fashion, and to express $\Phi^{(\mu)}(t)$ as the product of a factor $S(t)$ accounting for modulation and of a stochastic factor $F^{(\mu)}(t)$ accounting for the source intrinsic fluctuations in the form

$$
\Phi^{(\mu)}(t)=S(t) F^{(\mu)}(t),
$$

where it has been assumed, for the sake of simplicity, that all the sources $\mu$ are modulated identically. Furthermore, it is convenient to isolate in the expression of $P(t)$ the contributions that are due to the signal modulation by expanding $S\left(t^{\prime}\right)$ $=S\left[t-\left(L / V_{n}\right)\right]$ around $t^{\prime}=t$, that is,

$$
S\left(t-\frac{L}{V_{n}}\right)=S(t)-\frac{\mathrm{d} S(t)}{\mathrm{d} t} \frac{L}{V_{n}}+\ldots,
$$

thus getting, after inserting Eqs. (16) and (17) into Eq. (12),

$$
\begin{aligned}
P(t)= & M(t) \sum_{n} \sum_{k} \sum_{\mu} \sum_{\nu} \exp \left[i\left(\omega_{\mu}-\omega_{\nu}\right) t\right] \\
& \times \mathscr{F}_{n k}{ }^{(\mu, \nu)} A_{n}{ }^{(\mu)} A_{k}{ }^{(\nu) *} F^{(\mu)}\left(t-\frac{L}{V_{n}}\right) F^{(\nu) *}\left(t-\frac{L}{V_{k}}\right) \\
& +i M(t) \Omega(t) \sum_{n} \sum_{k} \sum_{\mu} \sum_{\nu} \exp \left[i\left(\omega_{\mu}-\omega_{\nu}\right) t\right] \\
& \times \mathscr{F}_{n k}{ }^{(\mu, \nu)} \tau_{k n} A_{n}{ }^{(\mu)} A_{k}{ }^{(\nu)^{*}} F^{(\mu)}\left(t-\frac{L}{V_{n}}\right) F^{(\nu)^{*}}\left(t-\frac{L}{V_{k}}\right) \\
& -\frac{1}{2} \frac{\mathrm{d} M(t)}{\mathrm{d} t} \sum_{n} \sum_{k} \sum_{\mu} \sum_{\nu} \exp \left[i\left(\omega_{\mu}-\omega_{\nu}\right) t\right] \\
& \times \mathcal{F}_{n k}{ }^{(\mu, \nu)}\left(\frac{L}{V_{n}}+\frac{L}{V_{k}}\right) A_{n}{ }^{(\mu)} A_{k}(\nu)^{*} F^{(\mu)} \\
& \times\left(t-\frac{L}{V_{n}}\right) F^{(\nu) *}\left(t-\frac{L}{V_{k}}\right)+\ldots,
\end{aligned}
$$

where we have defined $\tau_{k n}=\left(L / V_{k}\right)-\left(L / V_{n}\right), S(t)=$ $[M(t)]^{1 / 2} \exp [\operatorname{im}(t)]$, and $\Omega(t)=\mathrm{d} m / d t$.

The various terms appearing on the right-hand side of Eq. (18) are responsible for the linear and nonlinear distortions of the signal that are due to the fiber modal delays and are the analogs, in the time domain, of those introduced in Ref. 7 (to which the reader is referred for a quantitative analysis of their relevance in specific situations). We are concerned in the following only with the first one, which is dominant for small-bandwidth modulation (that is, for a signal-modulation time $\left.T_{M}>\left|\tau_{n k}\right|\right)$ and whose statistical behavior is representative also of the other terms. We can accordingly write 


$$
\begin{gathered}
P(t)=M(t) \sum_{n} \sum_{k} \sum_{\mu} \sum_{\nu} \exp \left[i\left(\omega_{\mu}-\omega_{\nu}\right) t\right] \\
\times \mathcal{F}_{n k}^{(\mu, \nu)} A_{n}{ }^{(\mu)} A_{k}{ }^{(\nu)^{*}} F^{(\mu)}\left(t-\frac{L}{V_{n}}\right) F^{(\nu)^{*}}\left(t-\frac{L}{V_{k}}\right) .
\end{gathered}
$$

\section{EVALUATION OF THE RELEVANT STATISTICAL QUANTITIES}

Before proceeding to perform the relevant averaging operations, it is necessary to recognize the presence, in Eq. (19), of various characteristic time scales. These are the mutual modal delays,

$$
\left|\tau_{k n}\right|=L\left|\frac{1}{V_{k}}-\frac{1}{V_{n}}\right|, \quad n \neq k
$$

the coherence times $T_{c}{ }^{(\mu)}$ of the various sources; the information modulation time $T_{M}$; the beating periods

$$
T^{\mu \nu}=\left|\frac{1}{\omega_{\mu}-\omega_{\nu}}\right|, \quad \mu \neq \nu
$$

the characteristic time $T_{E}$ of the fiber environment fluctuations; and finally, the time constant $T_{D}$ of the detector employed in the actual telecommunication link. Having limited ourselves to the analysis of the first term appearing on the right-hand side of Eq. (18), we have to limit our consideration to situations in which $\left|\tau_{n k}\right|<T_{M}$ [this ensures that the second term in Eq. (18) is small compared with the first]; in order to preserve the information, $T_{D}$ has to fulfill the requirement that $T_{D}<T_{M}$

We are concerned with two types of averaging. The first one is that which is performed in the actual operating communication system by the detector; we designate this averaging by \langle\rangle$_{D}$, quantity $\langle P(t)\rangle_{D}$ being proportional to the output signal from the detector. This quantity may, depending on the relative values of $T_{C}, T_{D}$, and $\left|\tau_{k n}\right|$, exhibit fluctuations caused by the limited coherence time of the source in addition to those connected with the variations of the fiber environment. In order to obtain a quantitative measure of these fluctuations and to evaluate their effect on the system performance, we can then calculate the variance of $\langle P(t)\rangle_{D}$ :

$$
\sigma^{2}=\frac{\left\langle\langle P(t)\rangle_{D}{ }^{2}\right\rangle-\left\langle\langle P(t)\rangle_{D}\right\rangle^{2}}{\left\langle\langle P(t)\rangle_{D}\right\rangle^{2}}
$$

where \langle\rangle represents an averaging operation over a time interval that is long compared with all the characteristic times of the power fluctuations in the fiber. It is, of course, to be understood that this second averaging is performed only to evaluate the system performance, and it is not actually carried out in the real communication system.

One needs first to compare the detection time $T_{D}$ with the coherence time $T_{C}$ of the exciting source, which is roughly the characteristic period of its fluctuations (in both the amplitude and the phase of the emitted radiation).

If $T_{D}$ is sensibly longer than $T_{C}$, the detector automatically performs a time average over these fluctuations, and the signal $\langle P\rangle_{D}$ at the output of the detector is deterministic but for the presence of the long-time-scale $\left(T_{E}\right)$ fluctuations associated with the fiber environment, which become the only source of noise. By indicating with \langle\rangle$_{f}$ the averaging operation over the fiber fluctuations, one then has

$$
\langle P\rangle \equiv\left\langle\langle P(t)\rangle_{D}\right\rangle=\left\langle\langle P(t)\rangle_{D}\right\rangle_{f}
$$

and

$$
\left\langle P^{2}\right\rangle \equiv\left\langle\langle P(t)\rangle_{D}^{2}\right\rangle=\left\langle\langle P(t)\rangle_{D}^{2}\right\rangle_{f}
$$

Furthermore, since $T_{C}$ is shorter than $T_{D}$, we can replace $\langle P\rangle_{D}$ with $\langle P\rangle_{s}$, where \langle\rangle$_{s}$ means a time average over a time interval long compared with the source fluctuation time (or ensemble average if one is dealing with a stationary random process).

If $T_{D}$ is sensibly shorter than $T_{C}$, a situation that may be encountered when one is dealing with a highly coherent radiation, the detector output signal contains the fluctuations that are due both to the exciting source and to the fiber environment. We can write in this case $\langle P(t)\rangle_{D}=P(t)$, so that one has

$$
\langle P\rangle \equiv\left\langle\langle P(t)\rangle_{s}\right\rangle_{f}
$$

and

$$
\left\langle P^{2}\right\rangle \equiv\left\langle\left\langle P^{2}(t)\right\rangle_{s}\right\rangle_{f}
$$

\section{Single Exciting Source}

\section{Case I}

Let us first examine the case of a single exciting source, and let us consider the situation in which

$$
T_{C}<T_{D}<T_{M}<T_{E}
$$

The detector automatically performs the averaging over the source fluctuations, and one has from Eq. (24) (omitting for simplicity the factor $M$ )

$$
\langle P\rangle_{s}=\sum_{n} \sum_{k} \mathcal{F}_{n k} A_{n} A_{k} *\left\langle F\left(t-\frac{L}{V_{n}}\right) F^{*}\left(t-\frac{L}{V_{k}}\right)\right)_{s} \cdot
$$

Thus, if one assumes that the stochastic process describing source fluctuations is a stationary one, the output signal fluctuations are due only to the slowly varying amplitudes $A_{k}$ 's. According to Eqs. (23) and (24), one has, in order to evaluate the system performance, to average $\langle P\rangle_{s}$ and its square

$$
\begin{aligned}
\left(\langle P\rangle_{s}\right)^{2}= & \sum_{n} \sum_{k} \sum_{n^{\prime}} \sum_{k^{\prime}} \mathscr{F}_{n k} \mathscr{F}_{n^{\prime} k^{\prime}} A_{n} A_{k} * A_{n^{\prime}} A_{k^{\prime}} * \\
& \times\left(F\left(t-\frac{L}{V_{n}}\right) F^{*}\left(t-\frac{L}{V_{k}}\right)\right)_{s} \\
& \times\left(F\left(t-\frac{L}{V_{n^{\prime}}}\right) F^{*}\left(t-\frac{L}{V_{k^{\prime}}}\right)\right)_{s}
\end{aligned}
$$

over the fiber fluctuations. Taking advantage of the fact that the relative phases of the $A_{n}$ 's are completely unpredictable, one has

$$
\begin{aligned}
\left\langle A_{n} A_{k} *\right\rangle_{f} & =\left\langle\left|A_{n}\right|^{2}\right\rangle_{f} \delta_{n k}, \\
\left\langle A_{n} A_{k} * A_{n^{\prime}} A_{k^{\prime}} *\right\rangle_{f} & =\left\langle\left|A_{n}\right|^{2}\left|A_{k}\right|^{2}\right\rangle_{f}\left(\delta_{n k} \delta_{n^{\prime} k^{\prime}}+\delta_{n k^{\prime}} \delta_{k n^{\prime}}\right),
\end{aligned}
$$

so that

$$
\langle P\rangle=\left\langle\langle P\rangle_{s}\right\rangle_{f}=\sum_{n} \mathcal{F}_{n n}\left\langle\left|A_{n}\right|^{2}\right\rangle_{f}
$$

and 


$$
\begin{aligned}
\left\langle P^{2}\right\rangle \equiv & \left\langle\left(\langle P\rangle_{s}\right)^{2}\right\rangle_{f}=\langle P\rangle^{2}+\sum_{n} \sum_{k} \mathscr{F}_{n n} \mathscr{F}_{k k}\left(\left\langle\left|A_{n}\right|^{2}\left|A_{k}\right|^{2}\right\rangle_{f}\right. \\
& \left.-\left\langle\left|A_{n}\right|^{2}\right\rangle_{f}\left\langle\left|A_{k}\right|^{2}\right\rangle_{f}\right)+\sum_{n} \sum_{\neq k} \mathfrak{F}_{n k} \mathfrak{F}_{k n} \\
& \times\left\langle\left|A_{n}\right|^{2}\left|A_{k}\right|^{2}\right\rangle_{f}\left|\left\langle F(0) F^{*}\left(\left|\tau_{n k}\right|\right)\right\rangle_{s}\right|^{2}
\end{aligned}
$$

where we have set, without loss of generality, $\left\langle|F(t)|^{2}\right\rangle_{s}=1$. The second and third terms on the right-hand side of Eq. (33) contribute to noise. The first of these is connected with the slow-mode-amplitude fluctuations that are due to the fiber environment and would also be present in a single-mode fiber; accordingly, it is not considered modal noise, for which only the last term is responsible. For simplicity we omit in the following the contribution that is due to the first kind of noise.

If the $\left|\tau_{n k}\right|$ 's exceed the coherence time of the source, $\left|\tau_{n k}\right|$ $>T_{C}$, then $\left\langle F(0) F^{*}\left(\left|\tau_{n k}\right|\right)\right\rangle_{s}=0$ and

$$
\left\langle P^{2}\right\rangle=\langle P\rangle^{2},
$$

which expresses the absence of modal noise. This fact also could have been guessed $a$ priori by inspecting Eq. (28) and by observing that the output signal does not contain any mode-interference terms $(n \neq k)$ if all the mutual modal delays exceed $T_{C}$.

\section{Case II}

Let us now consider the situation in which $T_{D}<T_{C}$, which applies in most single-mode semiconductor lasers whenever the modulation rate exceeds a few megahertz. In this case, since $T_{D}<T_{M}$, we also take $T_{M}<T_{C}$, so that the following hierarchy is satisfied:

$$
T_{D}<\left|\tau_{n k}\right|<T_{M}<T_{C}<T_{E}
$$

If the exciting source is a single-mode laser exhibiting only phase fluctuations, then

$$
F(t)=e^{i \phi(t)},
$$

where $\dot{\phi}$ represents the instantaneous frequency deviation $\delta \omega(t)$, and

$$
P(t)=\sum_{n} \sum_{k} \mathscr{F}_{n k} A_{n} A_{k} * \exp \left[i \delta \omega(t) \tau_{k n}\right] \simeq \sum_{n} \sum_{k} \mathscr{F}_{n k} A_{n} A_{k} *
$$

which no longer contains the source fluctuations since $\delta \omega \tau_{k n}$ $\sim \tau_{k n} / T_{C} \ll 1$. According to Eqs. (25) and (26), one has then to perform only the average over the fiber fluctuations, which immediately yields

$$
\langle P\rangle=\sum_{n} \mathscr{F}_{n n}\left\langle\left|A_{n}\right|^{2}\right\rangle_{f}
$$

and

$$
\left\langle P^{2}\right\rangle=\langle P\rangle^{2}+\sum_{n} \sum_{\neq k} \mathfrak{F}_{n k} \mathfrak{F}_{k n}\left\langle\left|A_{n}\right|^{2}\left|A_{k}\right|^{2}\right\rangle_{f},
$$

which coincide with Eqs. (32) and (33) whenever one assumes there that $\left|\tau_{n k}\right|<T_{C}$ and ignores the second term on the right-hand side of Eq. (33), as discussed above.

\section{Many Exciting Sources}

Let us now consider the case of many exciting laser sources. These can be, for example, the longitudinal modes of a single laser. Under the hypothesis that $T_{D}$ is larger than the largest beating period $T^{\mu \nu}$ (which, if the different sources were the longitudinal modes of a laser, would be the round-trip time of the cavity), the detector output signal is

$$
\begin{aligned}
P^{\prime}(t)= & \sum_{\mu} \sum_{n} \sum_{k} \mathscr{F}_{n k}{ }^{(\mu, \mu)} A_{n}{ }^{(\mu)} A_{k}{ }^{(\mu) *} F^{(\mu)} \\
& \times\left(t-\frac{L}{V_{n}}\right) F^{(\mu) *}\left(t-\frac{L}{V_{k}}\right),
\end{aligned}
$$

since all the nondiagonal terms average to zero. If we assume that all the $T_{C}{ }^{(\mu)}$ are shorter than $T_{d}$,

$$
T_{\mathcal{c}}(\mu)<T_{d}<T_{M}<T_{E},
$$

then one has

$$
\begin{aligned}
\left\langle P^{\prime}\right\rangle_{s}= & \sum_{\mu} \sum_{n} \sum_{k} \mathcal{F}_{n k}{ }^{(\mu, \mu)} A_{n}{ }^{(\mu)} A_{k}{ }^{(\mu)^{*}} \\
& \times\left\langle F^{(\mu)}\left(t-\frac{L}{V_{n}}\right) F^{(\mu)^{*}}\left(t-\frac{L}{V_{k}}\right)\right)_{s} .
\end{aligned}
$$

As in the case of the single source [see Eq. (28)], we can observe that, if the various exciting sources are stationary, the output signal depends on time only through the $A_{n}$ 's so the averages that one has to perform on $\left\langle P^{\prime}\right\rangle_{s}$ and on its square

$\left(\left\langle P^{\prime}\right\rangle_{s}\right)^{2}$

$$
\begin{aligned}
= & \sum_{\mu} \sum_{\nu} \sum_{n} \sum_{k} \sum_{n^{\prime}} \sum_{k^{\prime}} \mathscr{F}_{n k}{ }^{(\mu, \mu)} \mathscr{F}_{n^{\prime} k^{\prime}}{ }^{(\nu, \nu)} A_{n}{ }^{(\mu)} A_{k}{ }^{(\mu) *} A_{n^{\prime}}{ }^{(\nu)} A_{k^{\prime}}{ }^{(\nu) *} \\
& \times\left(F^{(\mu)}\left(t-\frac{L}{V_{n}}\right) F^{(\mu)^{*}}\left(t-\frac{L}{V_{k}}\right)\right)_{s} \\
& \times\left(F^{(\nu)}\left(t-\frac{L}{V_{n^{\prime}}}\right) F^{(\nu) *}\left(t-\frac{L}{V_{k^{\prime}}}\right)\right)_{s}
\end{aligned}
$$

are averages over the fiber fluctuations. By performing them, one obtains

$$
\begin{aligned}
& \langle P\rangle=\left\langle\left\langle P^{\prime}\right\rangle_{s}\right\rangle_{f}=\sum_{\mu} \sum_{n} \mathscr{F}_{n n}{ }^{(\mu, \mu)}\left\langle\left|A_{n}{ }^{(\mu)}\right|^{2}\right\rangle_{f} \\
\left\langle P^{2}\right\rangle= & \left\langle\left\langle P^{\prime}\right\rangle_{s}{ }^{2}\right\rangle_{f} \\
= & \langle P\rangle^{2}+\sum_{\mu} \sum_{n} \sum_{\neq k} \mathscr{F}_{n k}{ }^{(\mu, \mu)} \mathcal{F}_{k n}{ }^{(\mu, \mu)}\left\langle\left|A_{n}{ }^{(\mu)}\right|^{2}\left|A_{k}{ }^{(\mu)}\right|^{2}\right\rangle_{f} \\
& \times\left|\left\langle F^{(\mu)}(0) F^{(\mu)^{*}}\left(\left|\tau_{n k}\right|\right)\right\rangle_{s}\right|^{2}
\end{aligned}
$$

which can be compared with Eqs. (32) and (33). It is worthwhile to note that the basic assumption in deriving Eqs. (44) and (45) is not the statistical independence of the various sources but rather the fact that the phases of $A_{n}{ }^{(\mu)}$ and $A_{k}{ }^{(\nu)}$ are completely uncorrelated, that is,

$$
\begin{gathered}
\left\langle A_{n}{ }^{(\mu)} A_{k}{ }^{(\mu) *}\right\rangle_{f}=\left\langle\left|A_{n}{ }^{(\mu)}\right|^{2}\right\rangle_{f} \delta_{n k}, \\
\left\langle A_{n}{ }^{(\mu)} A_{k}{ }^{(\mu)^{*}} A_{n^{\prime}}{ }^{(\nu)} A_{k^{\prime}}{ }^{(\nu)^{*}}\right\rangle_{f}=\left\langle A_{n}{ }^{(\mu)} A_{k}{ }^{(\mu) *} A_{n^{\prime}}{ }^{(\nu)} A_{k^{\prime}}{ }^{(\nu)^{*}}\right\rangle_{f} \\
\times \begin{cases}\delta_{n k} \delta_{n^{\prime} k^{\prime}}+\delta_{n k^{\prime}} \delta_{k n^{\prime}} & \text { for } \mu=\nu \\
\delta_{n k} \delta_{n^{\prime} k^{\prime}} & \text { for } \mu \neq \nu\end{cases}
\end{gathered}
$$

This hypothesis may actually become questionable if the $\omega_{\mu}$ 's are not sufficiently spaced. 
As for the single source, we can now treat the situation

$$
T_{d}<\left|\tau_{n k}\right|<T_{M}<T_{C}^{(\mu)}
$$

by assuming that

$$
F^{(\mu)}(t)=\exp \left[i \phi_{\mu}(t)\right]
$$

where $\dot{\phi}_{\mu}=\delta \omega_{\mu}(t)$ represents the instantaneous frequency deviation of each exciting single-mode laser source. Also, in this case, in the quantity

$$
P^{\prime}(t)=\sum_{\mu} \sum_{n} \sum_{k} \mathcal{F}_{n k}{ }^{(\mu, \mu)} A_{n}{ }^{(\mu)} A_{k}{ }^{(\mu)^{*}} \exp \left(i \delta \omega_{\mu} \tau_{k n}\right),
$$

there is no trace left of the source fluctuations so that, averaging over the fiber, one gets

$$
\begin{aligned}
\langle P\rangle & =\sum_{\mu} \sum_{n} \mathscr{F}_{n n}{ }^{(\mu, \mu)}\left\langle\left|A_{n}{ }^{(\mu)}\right|^{2}\right\rangle_{f} \\
\left\langle P^{2}\right\rangle & =\langle P\rangle^{2}+\sum_{\mu} \sum_{n} \sum_{\neq k} \mathscr{F}_{n k}{ }^{(\mu, \mu)} \mathscr{F}_{k n}{ }^{(\mu, \mu)}\left\langle\left|A_{n}{ }^{(\mu)}\right|{ }^{2}\left|A_{k}(\mu)\right|^{2}\right\rangle_{f} .
\end{aligned}
$$

We can now observe, by inspecting Eqs. (44) and (45) or (51) and (52), that, since the quantities $\mathcal{F}_{n n}{ }^{(\mu, \mu)}$ and $\mathcal{F}_{n k}{ }^{(\mu, \mu)} \mathcal{F}_{k n}{ }^{(\mu, \mu)}$ do not depend on $\mu$ [see Eq. (11)], they are formally equivalent to the ones relative to the single-source excitation [Eqs. (28) and (29) or (32) and (33)], provided that the summation over the index $\mu$ is carried out first. In particular, the normalized variance

$$
\Sigma^{2}=\frac{\left\langle P^{2}\right\rangle-\langle P\rangle^{2}}{\langle P\rangle^{2}}
$$

takes, according to Eqs. (44) and (45), the form

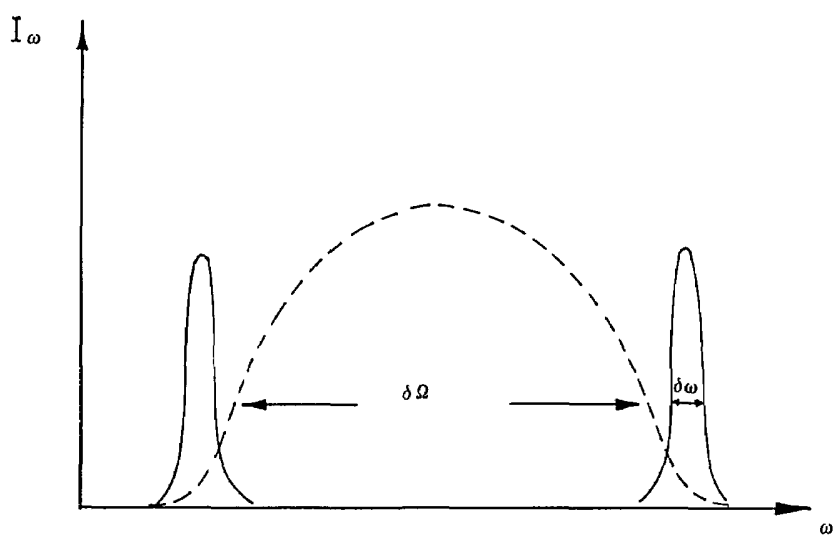

Fig. 2. Qualitative representation of the spectral intensity of the single large-bandwidth source (dashed line) and of the two narrowbandwidth sources.

It is instructive to show how the expressions for the variance furnished by Eqs. (54) and (56) permit one, without resorting to any specific model, to draw some conclusions on general questions such as the following: Is it more convenient, from the point of view of modal noise, to excite the fiber by means of a single source possessing a large bandwidth $\delta \Omega$ or by means of two narrow sources with bandwidth $\delta \omega$ (see Fig. 2)? If we assume that all the sources are similar and provide the same excitation, then Eq. (55) applies for $\tau_{n k}=0$, and the variance is reduced by a factor of 2 when two sources are employed; one has, however, to take into account that in this case the quantities $\left\langle F^{(\mu)}(0) F^{(\mu)^{*}}\left(\left|\tau_{n k}\right|\right)\right\rangle_{s}$ go to zero over a much larger distance $\left(\left|\tau_{n k}\right| \gg 1 / \delta \omega\right)$ than in the single-source case $\left(\left|\tau_{n k}\right|\right.$

$$
\Sigma^{2}=\frac{\sum_{n} \sum_{\not k} \mathscr{F}_{n k} \mathcal{F}_{k n} \sum_{\mu}\left\langle\left.\left|A_{n}{ }^{(\mu) \mid}{ }^{2}\right| A_{k}{ }^{(\mu)}\right|^{2}\right\rangle_{f}\left|\left\langle F^{(\mu)}(0) F^{(\mu) *}\left(\left|\tau_{n k}\right|\right)\right\rangle_{s}\right|^{2}}{\left(\sum_{n} \mathcal{F}_{n n} \sum_{\mu}\left\langle\left|A_{n}{ }^{(\mu)}\right|^{2}\right\rangle_{f}\right\rangle^{2}} .
$$

The expression of $\sum^{2}$ valid for Eqs. (51) and (52), $T_{D}<T_{L}$, is simply obtained from Eq. (54) by letting $\left|\tau_{n k}\right|$ go to zero. If we now assume that all the sources are similar and provide the same excitation into the fiber modes, then one has

$$
\Sigma^{2}=\frac{1}{N} \sigma^{2}
$$

where $N$ is the number of exciting sources and $\sigma^{2}$ is the normalized variance relative to the single-source excitation,

$$
\sigma^{2}=\frac{\sum_{n} \sum_{\neq k} \mathcal{F}_{n k} \mathcal{F}_{k n}\left\langle\left|A_{n}\right|^{2}\left|A_{k}\right|^{2}\right\rangle_{f}\left|\left\langle F(0) F^{*}\left(\left|\tau_{n k}\right|\right)\right\rangle_{s}\right|^{2}}{\left(\sum_{n} \mathcal{F}_{n n}\left\langle\left|A_{n}\right|^{2}\right\rangle_{f}\right)^{2}} .
$$

Equation (55) provides the basis for the statement that the performances of a connector are improved, as far as modal noise is concerned, by a factor $1 / \sqrt{N}$, thanks to the simultaneous excitation of the fiber by means of $N$ similar sources. $\gg 1 / \delta \Omega$ ), so that the choice between the two situations has to be made according to the length of fiber employed.

\section{CONCLUSIONS}

We have examined the statistical fluctuations arising in a signal that has propagated in a multimode fiber followed by an imperfect connector. These fluctuations are due to fluctuations in the exciting source as well as to those of the fiber parameters. The direct analysis in the time domain of the power coming out of the connector helps one to clarify the role played by the various time constants of the system when evaluating the relevant statistical averages necessary to characterize the process. In particular, we have worked out the general expression of the normalized variance of the signal when $N$ sources are simultaneously employed to excite the fiber; this allows us to show its $1 / N$ reduction, over the single-source case, whenever the sources are similar and excite the fiber in the same way.

* On leave of absence from Fondazione Ugo Bordoni, Roma, Italy. 


\section{REFERENCES}

1. B. Crosignani, B. Daino, and P. Di Porto, "Interference of mode patterns in optical fibers," Opt. Commun. 11, 178-179 (1974).

2. B. Crosignani, B. Daino, and P. Di Porto, "Speckle pattern visibility of light transmitted through a multimode fiber," J. Opt. Soc. Am. 66, 1312-1313 (1976).

3. R. E. Epworth, "The phenomenon of modal noise in analogue and digital fibre systems," in Proceedings of the Fourth European Conference on Optical Communication (Istituto Internazionale delle Comunicazioni, Genova, Italy, 1978), pp. 492-501.

4. B. Daino, G. De Marchis, and S. Piazzolla, "Analysis and measurement of modal noise in an optical fibre," Electron. Lett. 15,
755-756 (1979); "Speckle and modal noise in optical fibres. Theory and experiment," Opt. Acta 27, 1151-1159 (1980).

5. K. O. Hill, Y. Tremblay, and B. S. Kawasaki, "Modal noise in multimode fiber links: theory and experiment," Opt. Lett. 5, 270-272 (1980).

6. E. G. Rawson, J. W. Goodman, and R. E. Norton, "Frequency dependence of modal noise in multimode optical fibers," J. Opt. Soc. Am. 70, 968-976 (1980).

7. K. Petermann, "Nonlinear distortions and noise in optical communication systems due to fiber connectors," IEEE J. Quantum Electron. QE-16, 761-770 (1980).

8. B. Crosignani and P. Di Porto, "Soliton propagation in multimode optical fibers," Opt. Lett. 6, 329-330 (1981). 